\title{
Desarrollo de la competencia investigativa: una mirada desde el microcurrículo
}

\section{Development of investigative competence: a look from the microcurriculum}

\section{Olga Yanett Girón Márquez}

Candidata a Doctora en Educación con especialidad en Educación Superior Universidad de El Salvador olga.giron@ues.edu.sv https://orcid.org/0000-0003-3052-3921

\section{Referencia}

Girón Márquez, O. Y. (2021). Desarrollo de la competencia investigativa: una mirada desde el microcurrículo. Revista Guatemalteca de Educación Superior, 5(1), 160-170 . https://doi.org/10.46954/revistages.v5i1.83

\section{Resumen}

OBJETIVO: caracterizar fundamentos teóricos sobre el desarrollo de la competencia investigativa en la educación superior y la aplicación desde el microcurrículo. MÉTODO: se realizó una revisión documental, el estudio describe la definición del término competencia investigativa, su desarrollo, importancia y abordaje en la educación superior. RESULTADOS: una de las definiciones más completas de la competencia investigativa es la realizada desde el punto de vista del pensamiento complejo, las universidades han diseñado diferentes estrategias que les 
permite desarrollar dicha competencia. La postura de diferentes autores orienta que es el camino y el reto de las instituciones para dar respuesta a la formación investigativa de los profesionales que demanda la sociedad. CONCLUSIÓN: desde el microcurrículo el docente incide de forma directa en el proceso enseñanzaaprendizaje y puede contribuir con la formación integral y la mejora de la calidad institucional, a través del desarrollo de la competencia investigativa en el futuro profesional. Para potenciar dicha competencia el personal docente debe contar con los saberes, actitudes, valores y requerimientos necesarios para el diseño de la estrategia dentro del microcurrículo.

\section{Abstract}

OBJECTIVE: characterize theoretical foundations on the development of research competence in higher education and the application from the microcurriculum, METHOD: a documentary review was carried out, the study describes the definition of the term investigative competence, its development, importance and approach in higher education. RESULTS: one of the most complete definitions of investigative competence is the one made from the point of view of complex thinking, Universities have designed different strategies that allow them to develop this competence. The position of different authors guides what is the path and the challenge of the institutions to respond to the investigative training of professionals that society demands. CONCLUSION: from the microcurriculum, the teacher has a direct impact on the teaching-learning, and can contribute to comprehensive training and improvement of institutional quality, through the development of investigative competence in the future professional. To enhance this competence, the teaching staff must have the knowledge, attitudes, values and requirements necessary for the design of the strategy within the microcurriculum.

\section{Palabras clave:}

desarrollo de competencia, competencia investigativa, microcurrículo.

\section{Keywords:}

competence development, investigative competence, microcurriculum. 


\section{Introducción}

La sociedad demanda profesionales con una formación que les permita enfrentar situaciones de un entorno que se modifica constantemente. El desarrollo del componente investigativo en los estudiantes universitarios es fundamental, cumplir con este requerimiento se vuelve un reto para la universidad del siglo XXI, quienes deben asumir la formación investigativa. Cada institución de educación superior esta llamada a valorar el proceso formativo, estar abierta para adoptar nuevas concepciones pedagógicas y didácticas, acorde a los cambios que emergen.

A finales del siglo $X X$ e inicios del $X X I$ se incorpora el termino competencia en la educación formal y se retoma sin mayor pensamiento como concepto; al año 2021 han transcurrido más de dos décadas del uso del término en el área pedagógica. En la actualidad a nivel superior no todas las universidades cuentan con el conocimiento, las implicaciones y el abordaje del enfoque por competencias o se maneja un concepto reduccionista vinculado únicamente al desempeño laboral. De ahí la importancia de abordar la temática relacionada con el desarrollo de la competencia investigativa.

Al Considerar el contexto salvadoreño específicamente en la Universidad de El Salvador (UES), se valora la problemática sobre la formación investigativa que presenta cada estudiante con alto Coeficiente de Unidades de Mérito (CUM) honorífico, quien tiene la opción de graduarse automáticamente sin realizar proceso de grado. Además, se suman a esta realidad cada egresado que decide realizar una modalidad de proceso de grado desvinculada del quehacer investigativo. Situación que genera un vacío en el perfil del profesional y al reflexionar sobre la imposibilidad a corto plazo de realizar cambios o revisiones curriculares, surge la necesidad y la inquietud para encontrar una estrategia didáctica viable, oportuna, flexible y sostenida que posibilite el desarrollo de la competencia investigativa de los futuros profesionales. 
El ensayo tiene como objetivo caracterizar fundamentos teóricos sobre el desarrollo de la competencia investigativa en la educación superior y la aplicación desde el microcurrículo. La propuesta forma parte del resultado de la fundamentación teórica de la tesis doctoral: Modelo didáctico para el desarrollo de la competencia investigativa en estudiantes de la carrera de Licenciatura en Laboratorio Clínico de la UES, realizada por la autora del presente ensayo.

\section{Contenido}

\section{La competencia investigativa en la Educación Superior}

El futuro profesional debe estar debidamente formado para ir a la vanguardia de los cambios en la sociedad, la ciencia, tecnología e innovación. En la actualidad es necesario desarrollar las competencias para conferir idoneidad. La competencia investigativa forma parte del perfil de cada estudiante egresado, revisar la concepción de este término es fundamental para ser utilizado como enfoque en el ámbito universitario.

Para Pérez (2012) la competencia investigativa implica el saber utilizar el conocimiento para observar, preguntar, argumentar, sistematizar, con el propósito de generar conocimiento. Es de resaltar los aspectos: interés y motivación, el autor los considera parte del quehacer investigativo. Los aspectos referidos deben retomarse en el proceso educativo, desde la planificación, a efecto de dar garantía de la pasión por el objeto de estudio y la investigación misma.

Resulta muy interesante la sistematización teórica sobre competencias investigativas realizada por Estrada Molina (2014) quien revisó conceptos planteados por autores de Europa, Asia y América, un período de 15 años (1999-2013), prácticamente desde el surgimiento de este término compuesto. El autor analizó las dimensiones e indicadores en los conceptos propuestos para la formación, desarrollo y evaluación de ésta competencia, agrupándolos en cinco grupos. 
Para el primer grupo la competencia investigativa se conceptualiza como: Integración de dimensiones cognitivas, conocimientos y habilidades; cualidades personales, actitudes y destrezas; y metacognitivas que permiten al estudiante desempeñarse correctamente en una actividad de investigación. El segundo grupo, de acuerdo a la sistematización, además, de las dimensiones planteadas por el primer grupo, agrega otra dimensión: la profesional.

Mientras, el grupo tres incluye el aspecto motivacional y cualidades de personalidad. En concordancia con Pérez (2012), quién considera el interés y la motivación. Componentes fundamentales en este proceso formativo, los cuales ameritan prestar especial atención al momento de diseñar una estrategia. Así mismo el grupo cuatro incorpora la experiencia social del profesional como parte de la conceptualización para el desarrollo de la competencia investigativa.

La responsabilidad social es parte de la ética de todo profesional. Este componente, al igual que los mencionados por los grupos anteriores es tomado en cuenta por el quinto grupo de la clasificación realizada por Estrada Molina (2014) Es de resaltar el hecho que este grupo lo conforman autores que se identifican con el pensamiento complejo: Morín y Tobón entre otros. En este sentido la definición de la competencia investigativa es adoptada como un proceso complejo que permite un desempeño con idoneidad.

Si se pretende incidir en el desarrollo de la competencia investigativa considerando la definición que surge en el seno de los autores que manejan la complejidad, se debe pensar en el dominio de los diferentes saberes que implica esta concepción: saber ser, saber hacer, saber conocer y saber convivir en pro de la resolución de problemas. De acá la interrogante, ¿De qué manera la Institución de educación superior puede contribuir a mejorar su función formativa relacionada con la investigación? 
En tal sentido, Rodríguez Zambrano (2007) manifiesta que se requiere por parte de estas instituciones, agilizar el cambio del modelo de enseñanza al aprendizaje basado en competencias, incluyendo la evaluación. Por tanto, el diseño para desarrollar la competencia, debe considerar además de los componentes contemplados en la definición de la competencia investigativa bajo la mirada compleja, una estrategia de evaluación basada en competencias.

Por otra parte, en algunas universidades de América Latina incluyendo la Universidad de El Salvador, debido a diversas situaciones, prevalece una forma tradicional de la docencia. La actividad investigativa y la proyección social se encuentran desarticuladas, visualizándose la necesidad de abordar e incidir en el proceso de enseñanza, el cual debe estar acorde al profesional que se desea formar, en congruencia a la demanda social y contextual de la nación. En este marco, resulta indispensable reflexionar y tener claridad sobre la concepción de ser humano que se pretende formar, la finalidad esencial y la caracterización de este proceso de formación.

La Didáctica forma parte de las ciencias pedagógicas, el objeto de estudio de ella es el Proceso Enseñanza Aprendizaje (PEA), algunos autores, entre ellos Abreu, et al. (2018), especifican que el objeto no solamente se refiere al conocimiento de la estructura y funcionamiento del proceso, además, comprende lo que se quiere lograr a través de él. Este proceso según menciona Herrero y Valdés (2017) es intencional, planificado y creado, no espontáneo. Es de notar la relevancia de saber que se pretende lograr para estructurar una planificación estratégica que conduzca a tal fin.

Al concebir de esta manera las implicaciones del proceso enseñanza-aprendizaje, precisa una especial atención la formación investigativa que se ofrece en la educación superior. Es la Universidad que en cumplimiento de su misión y visión debe proyectarse a generar un impacto directo y positivo en el desarrollo, resultando indispensable replantear e incidir en este proceso. Los beneficios que se obtienen al desarrollar la 
competencia investigativa a nivel universitario, son tanto para la institución como para los profesionales, convirtiéndose en generadores de cambios que contribuyen al desarrollo de una región.

En este sentido, hay autores que reconocen el esfuerzo grande que implica para una universidad el sostener un equipo de investigación. Además, expresan la ventaja competitiva generada tanto para la institución como para los egresados. La garantía de un rendimiento académico y científico, favorece el descubrir y potenciar talentos como también generar cultura investigativa en estos ambientes formativos. Por tanto, se valora la importancia de alcanzar estas potencialidades.

\section{Desarrollo de la competencia investigativa desde el microcurrículo}

Uno de los compromisos sociales de la Universidad es formar profesionales que realicen investigación. Buscar las estrategias para asumir el reto de desarrollo de la competencia investigativa se vuelve fundamental para estas instituciones educativas. Cada carrera universitaria esta llamada a repensar la mejora en el proceso educativo, superar la desventaja cuando las directrices curriculares tardan en aparecer. El entorno cambia continuamente y requiere cambios en la formación de los profesionales.

En este sentido, es posible incidir desde el microcurrículo, para desarrollar acciones que solventen a corto plazo, los vacíos generados por la desactualización del currículo y la desvinculación con la formación investigativa que presentan diferentes universidades. La educación del siglo XXI demanda el desarrollo de competencias de manera integradora, enfocada al desarrollo de todos, según afirma Rodríguez et al. (2018). Además, este autor sostiene que la terminología competencia cada vez más se está integrando y aceptando en el mundo académico y científico. Por esta razón han surgido diferentes esfuerzos bajo esta lógica. 
Existen diversas estrategias empleadas por las universidades para potenciar la formación y desarrollar la competencia investigativa entre las cuales se pueden mencionar: semilleros de investigación, aprendizaje basado en proyectos, enfoques que consideran el proceso de enseñanza aprendizaje y algunos que utilizan la tecnología como es el caso del estudio Parra (2017) sobre los entornos con aprendizaje móvil con APPS.

Al considerar el contexto velozmente cambiante y dada su diversidad, se plantea la necesidad de brindar atención a los universitarios, bajo una nueva concepción de desarrollo de la competencia investigativa, con una mirada desde la epistemología de la complejidad, considerando un enfoque de integración. Así como lo plantea Tobón (2008), cuando describe el enfoque complejo de las competencias. Por otra parte, la pandemia por COVID-19 ha reactivado el reto investigativo en todas las profesiones, oportunidad para incentivar esta moción en el proceso formativo.

Diseñar una estrategia pedagógica que marque el camino de la Universidad hacia la sociedad del aprendizaje, incluyendo los diferentes saberes y las tecnologías de la información, es parte de los planteamientos de España Calderón (2020). Innovar en este sentido, y empezar a incidir desde el microcurrículo en las acciones academicas que forman parte del currículo y que estan relacionadas con el quehacer investigativo. Con la posibilidad de convertirse en estrategia de formación para los profesionales que demanda el siglo XXI.

Para incidir desde el microcurrículo es necesario valorar el perfil del docente a cargo del diseño. El docente debe poseer conocimientos sobre: didáctica con enfoque de competencias, la disciplina para el caso la metodología de la investigación, experiencia investigativa y el conocimiento del area laboral del futuro profesional que se esta formando. Además de estos saberes contar con cualidades, actitudes y valores que potencien la formación investigativa. Para luego crear el diseño de abordaje desde el microcurriculo con los elementos teoricos-prácticos que según Gonzalez (2012) lo integran, con el propósito formativo, desarrollo de la competencia investigativa. 


\section{Conclusión}

Las instituciones de educación superior en cumplimiento con la responsabilidad social están llamadas a realizar esfuerzos para garantía de la formación integral de los futuros profesionales que demanda el siglo XXI. El saber investigar es uno de los requerimientos del perfil de egreso. Sin embargo, al valorar la realidad de los egresados de la Universidad de El Salvador se ve la necesidad de buscar alternativas que abonen al desarrollo de la competencia investigativa.

La formación investigativa debe formar parte de las políticas de la Universidad. Diferentes autores concuerdan sobre la mejora de la calidad institucional y de formación a través del desarrollo de la competencia investigativa, por lo que instan a retomar este enfoque. La claridad de la concepción del término es fundamental, una definición muy acertada es la que se plantea a la luz del pensamiento complejo. Por analogía, el desarrollo de la competencia es complejo su definición y abordaje debe ser congruente a su naturaleza.

Incidir desde el microcurrículo, se vuelve una estrategia, posibilita al docente para trabajar a través del proceso enseñanzaaprendizaje. Esta estrategia permite superar la desventaja cuando se cuenta con un currículo desactualizado, y no tribute a algún componente de la formación requerida por el perfil de egreso. Así mismo, contribuye a la mejora de la calidad institucional y formativa. Por otra parte, es fundamental considerar el perfil del docente que pretende realizar transformaciones desde el microcurrículo. El personal docente a cargo debe contar con la capacitación, los saberes pertinentes, las cualidades, valores y actitudes propias para realizar el diseño que propicie y potencie el desarrollo de competencia, para este caso la investigativa. 


\section{Referencias}

Abreu, O., Rhea, S., Arciniegas, G., \& Rosero, M. (2018). Objeto de Estudio de la Didáctica: Análisis Histórico Epistemológico y Crítico del Concepto. Formación Universitaria, 11(6), 75-82. https://dx.doi.org/10.4067/S0718-50062018000600075

España Calderón, O. (2020). Globalización y tecnologías de la información: Impacto en la Universidad. Guatemala: Óscar de León Palacios. (impreso)

Estrada Molina, O. (2014). Sistematización teórica sobre la competencia investigativa. Revista electrónica Educare, 18(2), 177-194.http://www.scielo.sa.cr/scielo.php?script=sci_ arttext\&pid=S1409-42582014000200009\&lng=en\&tlng=es.

González Garcia, E. A. (2012). Fundamento teórico y metodológico del microcurrículo. Universidad Francisco de Paula SantanderCÚCUTA. CD-ROM.

Herrero, E., \& Valdés, N. (2017). Problemas actuales de la pedagogía y la formación del profesional universitario. La Didáctica en el contexto de las ciencias Pedagógicas. En R. Collazo, \& E. Herrero, Preparación Pedagógica para profesores de la nueva Universidad Cubana (págs. 3-19). Editorial Universitaria Felix Varela. http://profesorailianartiles.files.wordpress. com/2013/03/libro-de-preparacic3b3n-pedagc3b3gicaintegral.pdf

Parra, C. (2017). Formacion investigativa en estudiantes de pregrado mediantes entornos de aprendizaje móvil con APPS. EDUTEC Revista Electrónica de Tecnología Educativa(61), 1-14. https://doi.org/10.21556/edutec.2017.61.929

Pérez, M. (2012). Fortalecimiento de las competencias investigativas en el contexto de la Educación Superior en Colombia. Investigacion UNAD, 01, 9-34. https://doi. org/10.22490/25391887.770

Rodríguez Zambrano, H. (junio de 2007). El paradigma de las competencias hacia la educación superior. Revista Facultad de Ciencias Económicas: Investigación y Reflexión, XV(1), 145165. https://www.redalyc.org/articulo.oa?id=90915108 
Rodriguez, J., navarrete, Y., \& Holguin, R. (2018). Una didaáctica para el desarrollo de las competencias investigativas del profesional en formación inicial y permanente. Revista Cubana de Educación Superior(1), 162-170. Obtenido de http://scielo.sld.cu/scielo.php?script=sci_ isoref\&pid=S0257-43142018000100012\&lng=es \&tlng=es\#: :text=http\%3A//scielo.sld.cu/scielo. php\%3Fscript\%3Dsci_arttext\%26pid\%3DS025743142018000100012\%26lng\%3Des\%26tlng\%3Des.

Sancho Gil, J., \& Correa, G. (2010). Cambio y continuidad en sistemas educativos en transformación. Revista de Educación(352), 17-21. Obtenido de https://www. researchgate.net/publication/44204803_Cambio_y_ continuidad_en_sistemas_educativos_en_transformacion

\section{Sobre la autora}

Olga Yanett Girón Márquez, Licenciada en Química y Farmacia, con maestría en Administración de Empresas y maestría en Métodos y Técnicas de Investigación Social, candidata a doctora en Educación con especialidad en Educación Superior. Actualmente coordina un equipo multidisciplinario de investigación en el área de la salud en la Facultad Multidisciplinaria Oriental, Universidad de El Salvador.

\section{Conflicto de intereses}

Declara no tener ningún conflicto de intereses.

\section{Declaración de consentimiento informado}

El estudio se realizó con fines educativos, respetando el Código de ética y buenas prácticas editoriales de publicación.

\section{Derechos de uso \\ Copyright@ 2021 por Olga Yanett Girón Márquez \\ Este texto está protegido por la Licencia Creative Commons Atribución 4.0 Internacional.}

Usted es libre para compartir, copiar y redistribuir el material en cualquier medio o formato y adaptar el documento, remezclar, transformar y crear a partir del material para cualquier propósito, incluso comercialmente, siempre que cumpla la condición de atribución: usted debe reconocer el crédito de una obra de manera adecuada, proporcionar un enlace a la licencia, e indicar si se han realizado cambios. Puede hacerlo en cualquier forma razonable, pero no de forma tal que sugiera que tiene el apoyo del licenciante o lo recibe por el uso que hace. 